\title{
ARTICLES
}

\section{Global trends of whole-genome duplications revealed by the ciliate Paramecium tetraurelia}

Jean-Marc Aury ${ }^{1 \star}$, Olivier Jaillon ${ }^{1 \star}$, Laurent Duret ${ }^{2}$, Benjamin Noel $^{1}$, Claire Jubin ${ }^{1}$, Betina M. Porcel ${ }^{1}$, Béatrice Ségurens ${ }^{1}$, Vincent Daubin ${ }^{2}, V_{\text {éronique Anthouard }}{ }^{1}$, Nathalie Aiach $^{1}$, Olivier Arnaiz ${ }^{3}$, Alain Billaut ${ }^{1}$, Janine Beisson ${ }^{3}$, Isabelle Blanc ${ }^{3}$, Khaled Bouhouche ${ }^{4}$, Francisco Câmara ${ }^{5}$, Sandra Duharcourt ${ }^{4}$, Roderic Guigo ${ }^{5}$, Delphine Gogendeau ${ }^{3}$, Michael Katinka ${ }^{1}$, Anne-Marie Keller ${ }^{3}$, Roland Kissmehl ${ }^{6}$, Catherine $\mathrm{Klotz}^{3}$, France Koll ${ }^{3}$, Anne Le Mouël ${ }^{4}$, Gersende Lepère ${ }^{4}$, Sophie Malinsky ${ }^{4}$, Mariusz Nowacki ${ }^{4}$, Jacek K. Nowak ${ }^{7}$, Helmut Plattner ${ }^{6}$, Julie Poulain ${ }^{1}$, Françoise Ruiz ${ }^{3}$, Vincent Serrano ${ }^{4}$, Marek Zagulski ${ }^{7}$, Philippe Dessen ${ }^{8}$, Mireille Bétermier ${ }^{3,4}$, Jean Weissenbach ${ }^{1}$, Claude Scarpelli ${ }^{1}$, Vincent Schächter ${ }^{1}$, Linda Sperling ${ }^{3}$, Eric Meyer ${ }^{4}$, Jean Cohen ${ }^{3}$ $\&$ Patrick Wincker ${ }^{1}$

The duplication of entire genomes has long been recognized as having great potential for evolutionary novelties, but the mechanisms underlying their resolution through gene loss are poorly understood. Here we show that in the unicellular eukaryote Paramecium tetraurelia, a ciliate, most of the nearly 40,000 genes arose through at least three successive whole-genome duplications. Phylogenetic analysis indicates that the most recent duplication co incides with an explosion of speciation events that gave rise to the $P$. aurelia complex of 15 sibling species. We observed that gene loss occurs over a long timescale, not as an initial massive event. Genes from the same metabolic pathway or protein complex have common patterns of gene loss, and highly expressed genes are over-retained after all duplications. The conclusion of this analysis is that many genes are maintained after whole-genome duplication not because of functional innovation but because of gene dosage constraints.

Ciliates are unique among unicellular organisms in that they separate germline and somatic functions ${ }^{1}$. Each cell harbours two kinds of nucleus, namely silent diploid micronuclei and highly polyploid macromuclei. The latter are unusual in that they contain an extensively rearranged genome streamlined for expression and divide by a non-mitotic process. Only micronuclei undergo meiosis to perpetuate genetic information; the macronuclei are lost at each sexual generation and develop anew from the micronuclear lineage.

In Paramecium the exact number of micronuclear chromosomes (more than 50) and the structures of their centromeres and telomeres remain unknown. During macronuclear development, these chromosomes are amplified to about 800 copies and undergo two types of DNA elimination event. Tens of thousand of short, unique copy elements (internal eliminated sequences) are removed by a precise mechanism that leads to the reconstitution of functional genes ${ }^{2}$.

Transposable elements and other repeated sequences are removed by an imprecise mechanism leading either to chromosome fragmentation and de novo telomere addition or to variable internal deletions $^{3}$. These rearrangements occur after a few rounds of endoreplication, leading to some heterogeneity in the sequences abutting the imprecisely eliminated regions ${ }^{3}$. The sizes of the resulting, acentric macronuclear chromosomes range from 50-1,000 kilo- bases (kb) as measured by pulsed-field gel electrophoresis. Because the sexual process of autogamy results in an entirely homozygous genotype ${ }^{4}$, the macronuclear DNA that was sequenced was genetically homogeneous.

\section{The Paramecium genome sequence}

The Paramecium macronuclear genome sequence was established with the use of a whole-genome shotgun and assembly strategy. Paired-end sequencing of plasmid and bacterial artificial chromosome (BAC) clones provided a coverage of 13 genome equivalents (Supplementary Table S1). We assembled the sequence reads with Arachne $e^{5}$ in 1,907 contigs connected in 697 scaffolds of size greater than $2 \mathrm{~kb}$, giving a total coverage of $72 \mathrm{Mb}$. Half of the assembly is contained in 64 scaffolds larger than $413 \mathrm{~kb}$ (the N50 size), and $96 \%$ of the assembly $(69 \mathrm{Mb})$ in 188 scaffolds larger than $45 \mathrm{~kb}$. A majority of the 188 largest scaffolds are complete macronuclear chromosomes, as determined by analysis of telomere repeats, and on average they contain one gap every $120 \mathrm{~kb}$ with a mean estimated size of $0.66 \mathrm{~kb}$ (Supplementary Table S2). The largest scaffold, $981 \mathrm{~kb}$ long, corresponds to the largest chromosome observed by pulsed-field analysis. Consistent with the estimated gap frequency and sizes, alignment of this scaffold with the finished, independently determined, reference 
sequence of that chromosome ${ }^{6}$ revealed only four small gaps (less than 250 bases), and less than one difference per $6 \mathrm{~kb}$. If this chromosome is representative, then the present assembly contains more than $99 \%$ of the genome sequence with more than $99.95 \%$ accuracy.

The Paramecium macronuclear genome has a composition of $28 \%$ $\mathrm{G}+\mathrm{C}$ and is devoid of highly repeated sequences (Supplementary Figure $\mathrm{S1}$ ). The CpG dinucleotide frequency is severely depressed ( 0.42 -fold the expected value) and this cannot be accounted for by codon usage (Supplementary Table S3). In plant and vertebrate genomes, CpG depression is thought to be a consequence of cytosine methylation, but cytosine methylation has never been observed in Paramecium.

The genome sequence was annotated by using a combination of $a b$ initio predictions, comparative genomics and complementary DNA alignments. A specific resource of about 90,000 expressed sequence tags (ESTs) from six physiological states or developmental stages of Paramecium was generated for this purpose. The current proteincoding catalogue is composed of 39,642 genes (Table 1). About $55 \%$ of predicted Paramecium proteins have no currently referenced InterPro domain, a surprisingly high percentage that probably reflects the great evolutionary distance between Paramecium and other eukaryotic genomes sequenced so far. The macronuclear genome is compact ( $78 \%$ coding density), with extremely small intergenic regions ( 352 bases on average) and introns ( $25 \mathrm{bp}$ on average). Remarkably, in contrast with other compact genomes, Paramecium genes contain many introns and have an intron/exon ratio closer to that of some invertebrates such as D. melanogaster than to that of other sequenced unicellular eukaryotes (Table 1). When looking at the ESTs mapped to each annotated gene, we found no evidence of exon skipping. Adaptation of the splicing machinery to the reduced intron sizes may be incompatible with alternative splicing.

The ciliate clade is generally considered to have evolved from an ancestor with a red algal endosymbiont that gave rise to all extant species of the chromist and alveolate groups (the 'chromalveolate' hypothesis) ${ }^{7}$. We failed to find any clear case of genes characteristic of the algal lineage, or of genes of chloroplastic origin (see Supplementary Information). This implies that ciliates may have lost all traces of this ancient event because they no longer need photosynthesis or other algal-associated functions. Alternatively, endosymbiosis might not have occurred at the root of the chromalveolate lineage. In this hypothesis, the plastid-derived genes observed in apicomplexan parasites may be the result of a symbiotic event that occurred after their separation from the ciliate lineage.

A surprising result of the annotation process was the prediction of 39,642 protein-coding genes, a number unlikely to be an artefact of the gene prediction procedure (see Supplementary Table S5). P. tetraurelia is among the most gene-rich organisms yet sequenced, including many metazoans and plants. Using all-against-all comparisons, we observed that many of the protein-coding genes in Pa ramecium are present in closely related gene families (not shown). This redundancy may explain the high gene number.

\section{Genome duplications}

We aligned each protein against the whole proteome to identify duplicated genes (see Methods). This yields a complete picture of a whole-genome duplication (WGD) linking two by two all of the 188 large scaffolds (example in Fig. 1 and global view in the outer circle of Fig. 2).

One remarkable characteristic of this WGD is the conservation of synteny: only 8 translocations and 76 intemal inversions of chromosomal segments could be deduced. We found 10 instances in which two scaffolds ending in telomeres were paired with a single, longer scaffold, indicating that the germline fragmentation regions (for example transposons) might have moved since the WGD. However, validation of this will require examination of the corresponding micronuclear regions, because DNA elimination events that lead to chromosome fragmentation can also resolve to internal deletions. A second striking feature of this recent duplication is the number of genes retained in duplicate. About $68 \%$ of the proteome is composed of two-gene families originating from this event, whereas only $32 \%$ corresponds to genes returned to a single state by the loss of one of the two paralogues. This translates into $51 \%$ of the preduplication genes still present in two copies, corresponding to 12,026 ancestral genes, a number far greater than for any previously studied WGD. The recent Paramecium WGD thus contrasts with the WGDs discovered in yeast, fish or higher plants in recent years, in which synteny, or duplicate gene conservation, is greatly eroded and the WGD events could not always be detected without comparison with a non-duplicated reference genome $\mathrm{e}^{8-17}$.

All Paramecium chromosomes seem to be conserved after the duplication, and the 11,451 genes lost since the WGD seem to have disappeared through local decay mechanisms acting on a single gene or small region (Supplementary Fig. S11). The fact that we could detect a wide range of decay states (Supplementary Table S8 and Supplementary Fig. S12), indicates that pseudogenization might not have occurred abruptly after the WGD event, and we cannot exclude the possibility that some of these regions are still present in the micronuclear genome but are no longer amplified during macronuclear development ${ }^{18}$. We estimate the number of recent pseudogenes present in the annotated genes to be about 1,500 (see Supplementary Information).

We inferred the ancient gene order on the basis of the actual chromosomes (see Methods), revealing that two other WGD events had occurred before the recent WGD (Fig. 2, second and third circles

Table 1 | Comparison of $\boldsymbol{P}$. tetraurelia gene character istics with selected sequenced eukaryotes

\begin{tabular}{|c|c|c|c|c|c|c|c|c|c|c|}
\hline \multirow{3}{*}{$\begin{array}{l}\text { Cladistic } \\
\text { Species }\end{array}$} & \multirow{2}{*}{\multicolumn{2}{|c|}{$\begin{array}{c}\text { Chromalveolates } \\
\text { Alveolates }\end{array}$}} & \multicolumn{2}{|c|}{ Plantae } & \multicolumn{6}{|c|}{ Unikonts } \\
\hline & & & \multirow{2}{*}{$\begin{array}{c}\text { Red algae } \\
\text { Cyanidiophytes } \\
\text { C. merolae† }\end{array}$} & \multirow{2}{*}{$\begin{array}{l}\text { Streptophytes } \\
\text { Land plants } \\
\text { A. thaliana* }\end{array}$} & \multirow{2}{*}{$\begin{array}{l}\text { Amoebozoa } \\
\text { Dictyostelids } \\
\text { D. discaideum* }\end{array}$} & \multicolumn{5}{|c|}{ Opisthok onts } \\
\hline & $\begin{array}{l}\text { Cilliates } \\
\text { P. tetrourelia }\end{array}$ & $\begin{array}{l}\text { Apicomplexa } \\
\text { P. falciparum }\end{array}$ & & & & $\begin{array}{l}\text { Asconycetes } \\
\text { S. cerevisioes }\end{array}$ & C. elegans I| & $\begin{array}{c}\text { Animal } \\
\text { D. melanogastert }\end{array}$ & T. nigrovirids Sी & H. sapiens" \\
\hline Number of genes & 39,642 & 5,268 & 5,331 & 25,498 & 12,500 & 5,857 & 21,003 & 14,399 & 23,000 & 22,287 \\
\hline $\begin{array}{l}\text { Mean coding size } \\
\text { (amino acids) }\end{array}$ & 454 & 761 & 517 & 437 & 518 & 497 & 441 & 572 & 405 & 509 \\
\hline $\begin{array}{l}\text { Genes with introns } \\
\text { (\%) }\end{array}$ & 80 & 54 & 0.5 & 79 & 69 & 5.2 & 93 & 78 & 81 & 85 \\
\hline $\begin{array}{l}\text { Mean intron size } \\
\text { (bp) }\end{array}$ & 25 & 179 & 248 & 170 & 146 & 207 & 445 & 1,558 & 568 & 3,365 \\
\hline $\begin{array}{r}\text { Mean no. of introns } \\
\text { (in spliced genes) }\end{array}$ & 29 & 26 & 1.0 & 5.4 & 1.9 & 1.0 & 5.1 & 3.7 & 7.3 & 8.1 \\
\hline
\end{tabular}

"Most data are from ref. 45 .

$\uparrow$ According to ref. 46 .

\pm Computed with BDGP4 annotation reported at ensemb134.

$\$$ Camputed with SGD1 annotation reported at ensemb134.

1 Camputed with CEL 150 annotation reported at ensembl34.

TEstimated value from ret 13 . 


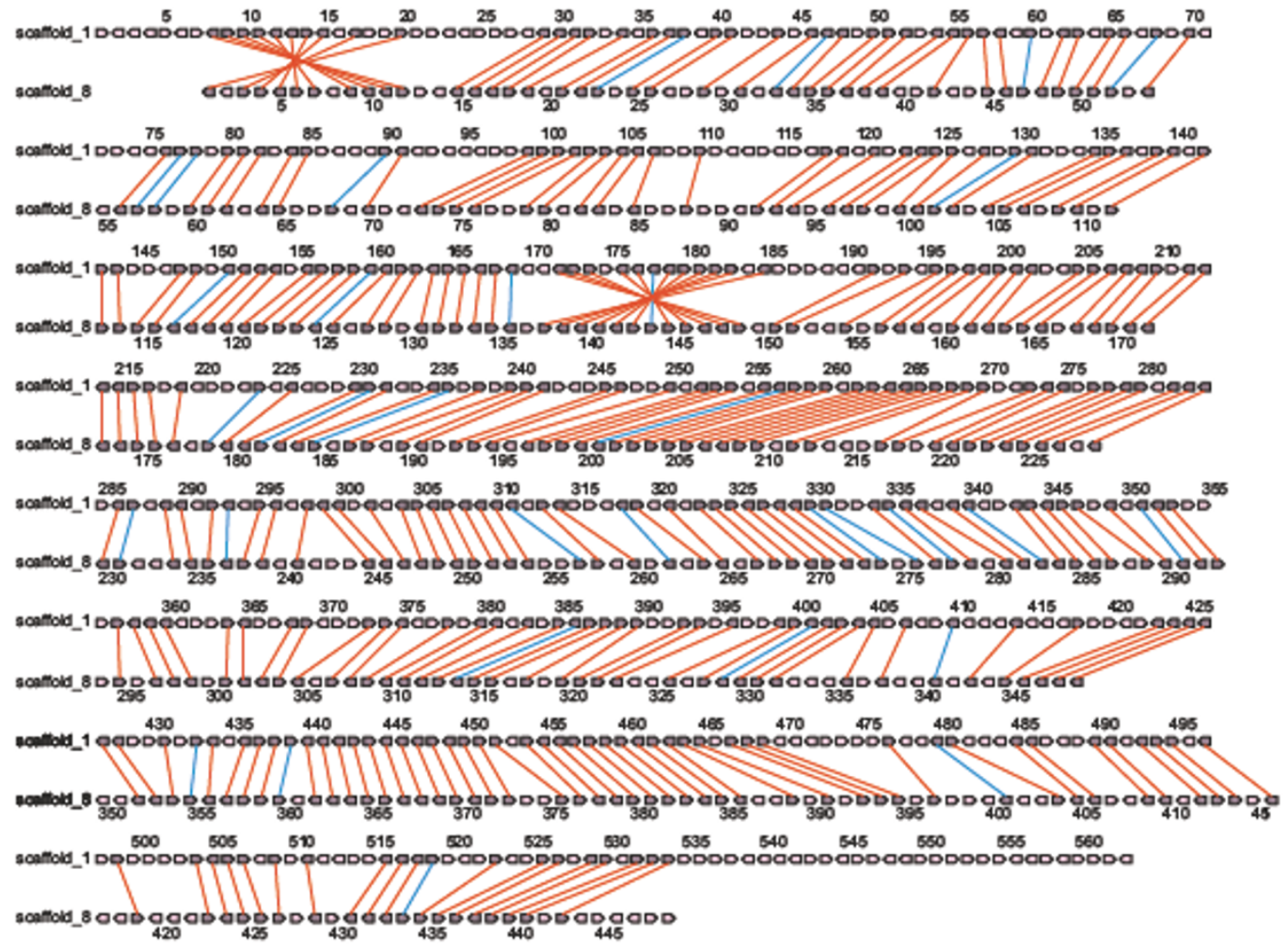

Figure 1 | Comparison of two scaffolds originating from a common ancestor at the recent WGD. Each arrow represents one gene with its direction of transcription. Brown arrows are genes with a detected paralogue, and pink arrows are genes with no paralogue. Paralogy relations are indicated by a solid line, red for genes with a BRH match, and blue for genes with a non-BRH syntenic match. Genes at the telomeric ends have paralogues scattered between different chromosomes, probably as a result of frequent recombination. from the outside). In comparison with the recent WGD, the number of genes conserved is smaller, as is the average similarity between paralogues (Table 2 and Fig. 3). We calculate the number of protein-coding genes in the ancestral genome before the three WGD events to be 19,552 by extrapolation of the number of genes present in the paralogons (paralogons are pairs of paralogous blocks that could be recognized as deriving from a common ancestral region). Therefore, taken together, the three WGDs have led to an approximate doubling of the gene count, explaining most of the gene number paradox in $P$. tetraurelia.

Application of the reconstruction and matching method a third time revealed a significant number of duplicated blocks with 436 duplicated genes (Fig, 2, innermost circle), and yet lower similarity between paralogues (Fig. 3a). It is therefore probable that the Paramecium lineage underwent a fourth, more ancient, WGD. The paralogous genes obtained in the final round of reconstruction, near detection limits, are called 'ancient duplicates', with no assumption as to their origin.

Nucleardimorphism in Paramecium may have helped in tolerating WGD, because the ploidy of the expressed macronucleus is subject to physiological regulation to maintain a constant nucleocytoplasmic ratio $^{19}$; it is therefore possible that a sudden duplication of the micronuclear genome does not alter macronuclear ploidy. However, it is not clear whether this influences the recurrent appearance of WGDs, which have been found in other taxa without nuclear dimorphism, 9.

\section{Retention and evolution of duplicate genes}

The nature of the duplicated genes that are retained or not after a WGD can shed light on the still debated forces shaping the genome in the wake of these drastic molecular events ${ }^{20}$. Most studied WGDs are ancient and have provided clues about their long-term effects. In particular, over-retention of signalling molecules and transcription factors has been observed, linking WGD with the evolution of regulatory complexity ${ }^{21,22}$. Short-term effects have not been easy to track. Very different theories on the forces that drive the retention or loss of gene duplicates include buffering for essential genes, enhancement of metabolic fluxes, protein dosage effects and rapid divergence of gene pairs ${ }^{23-28}$. Because these models predict different retention outcomes according to gene classes, we analysed the predicted gene categories in Paramecium for their propensity for duplicate retention.

The relative expression levels of proteins inwolved in the same complex (or metabolic pathway; see below) must be balanced to

Table 2 | Gene content of the paralogons for each duplication

\begin{tabular}{|c|c|c|c|c|c|}
\hline Duplication event & Genes in par alogans" & $\begin{array}{l}\text { Ancestral genes } \uparrow \\
\text { in par alogons }\end{array}$ & $\begin{array}{l}\text { Ancestral genes } \\
\text { with paralogue(s) }\end{array}$ & $\begin{array}{l}\text { Ancestr al genes } \\
\text { without par alogue }\end{array}$ & $\begin{array}{c}\text { Percentage of genes in the } \\
\text { reconstruction! }\end{array}$ \\
\hline Recent WGD & 35,503 & 35,503 & 24,052 & 11,451 & 90 \\
\hline Intermediary WGD & 31,129 & 20,578 & 7,996 & 12,582 & 79 \\
\hline Old WGD & 18,792 & 9,999 & 1,530 & 8,469 & 47 \\
\hline Ancient duplicates & 9,735 & 4,830 & 436 & 4,394 & 25 \\
\hline
\end{tabular}

*All genes present in a paralogan, whether a duplicated copy is retained or not.

$\uparrow$ An ancestral gene is a gene present before a given duplication that could be represented by ane or two retained par alogous copies.

t Percentage of genes from the annotated genome present in the ancestral blocks reconstructed from the paralogous rel ationships. 
ensure proper stoichiometry within the complex ${ }^{24}$. Thus, if duplicated genes are involved in a protein complex, the loss of one gene copy is expected to be counterselected unless it is compensated for by the upregulation of the other copy or by gene losses of other partners. To test this hypothesis we defined Paramecium orthologues of Saccharomyces cerevisiae genes involved in known complexes (Comprehensive Yeast Genome Database (CYGD) on the MIPS website (http://mips.gsf.de/), and ref. 29). Because we retained only clear orthologues between the two species, we expect most of the detected proteins to have ancient conserved functions and therefore a high probability of belonging to conserved complexes. We observed a strong over-retention of genes from the recent WGD involved in known complexes (Fig. 4a). The absence of any common functional theme among the complexes analysed here makes it unlikely that there has been enrichment of specific functions. In contrast, the copy number of each protein within a complex is significantly correlated with that of the other components of the complex ( $P$-value details are given in Supplementary Tables S9 and S10). We concluded that stoichiometry of the proteins constituting most complexes tends to be conserved. It has been previously shown that imbalance in the

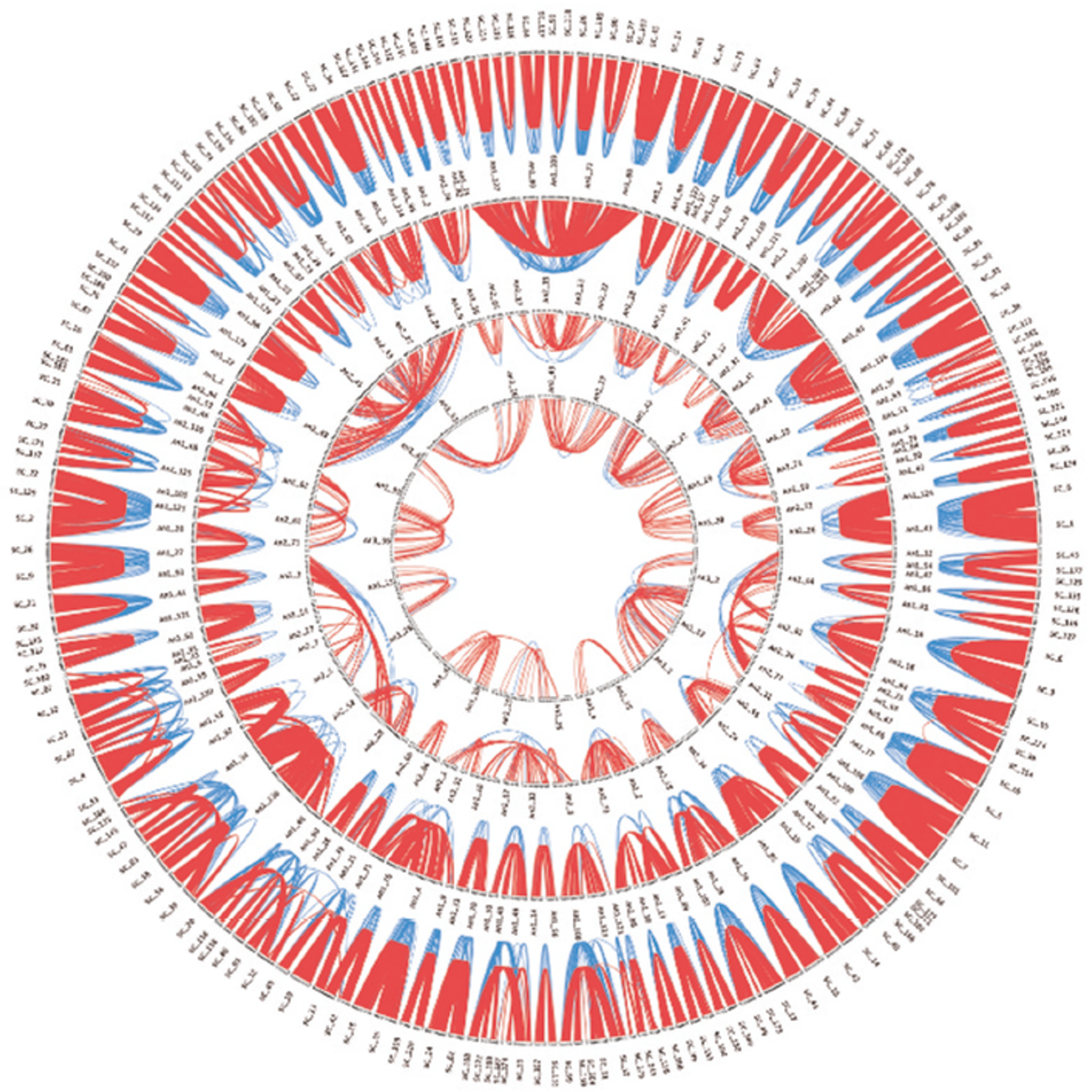

Figure 2 | Representation of the successive duplications of the Paramecium genome. The exterior cirde displays all hromosome-sized scaffolds, and the three interior circles show the reconstructed sequences obtained by fusion of the paired sequences from each previous step. Red lines link pairs of genes with a BRH match, and blue lines link pairs of genes with a non-BRH match that were added on the basis of syntenic position. Only the BRHs that link two genes in the same paralogon are represented. The position of an ancestral block is unrelated to the position of its constituents in the previous circle. 
a

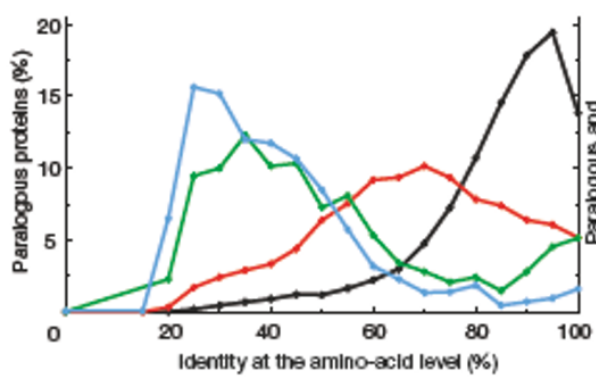

Figure 3 | Percentage identity between par alogous proteins, and comparisons with inter-species distances. a, Percentage identity is plotted as a function of the percentage of cases for paralogues originating from the recent (black), intermediary (red) and old (green) WGDs and from the

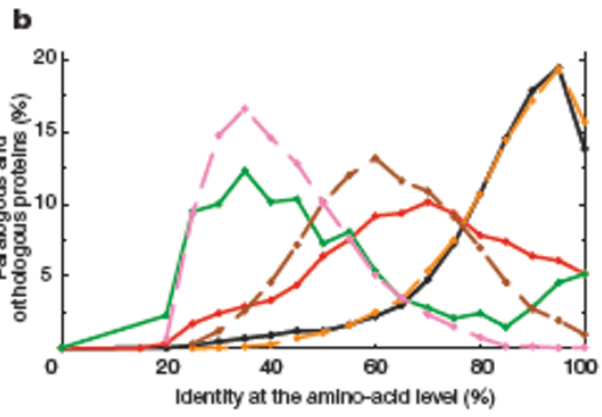

ancient duplicates (blue), b, Comparison of the three WGD plots with the distribution of orthologous matches between human and mouse (orange broken line), between human and the fish Tetraodon (brown broken line), and between Paramecium and Tetrahymena thermophila (pink broken line). concentration of complex components can frequently be harmful ${ }^{24}$. A key function for stoichiometry in this covariation is supported by two observations. First, the conservation of gene duplicates in a complex sometimes arises from the retention of genes originating from different duplication events, resulting in identical copy numbers for each protein (Supplementary Table S11). Second, proteins involved in many different complexes are highly retained in the recent WGD but are completely absent as retained duplicates from the old WGD (Supplementary Fig. S20). This indicates that gene loss for complex constituents might be a two-step process, in which proteins with many interactions are preferentially lost as more genes are inactivated, after a first period of preferential conservation to maintain the initial stoichiometry.

A striking result came from an analysis of central metabolism genes, which show a clear decline in retention over time. All central pathways evolve with the same trend, although at different rates (Fig. 4b). Most pathways are still significantly overamplified with respect to the recent duplication, an effect that has not been observed in WGDs analysed in other organisms ${ }^{21.30}$. As with protein complexes, the genes involved in a common metabolic pathway show a tendency to covary across the duplications, behaviour that can probably be attributed to stoichiometry, because imbalance in metabolic pathways may have a similar negative effect to that in multiprotein complexes.
The loss of one paralogue would be a slow process, involving overexpression of the other copy to maintain stoichiometric constraints.

A strong correlation is observed between expression levels and retention rates of WGD paralogues across all duplications (Fig. 4c). When we consider only the proteins previously defined as associated within complexes, the level of retention is greater (Supplementary Fig. S22), showing that retention is affect ed both by expression levels and by association in complexes. High expression is therefore a genuine effect in duplicate retention, whether or not the protein belongs to a complex. This correlation has already been observed on a smaller scale in the yeast genome duplication ${ }^{30}$, indicating that it might be a general trait of WGD.

We noticed the existence of a small group of paralogous genes that retained high expression levels, low amino-acid replacement rates and low rates of silent substitution of nucleotides. These genes also have biased codon usage (Supplementary Fig. S21), with many codons apparently optimized for high expression, because their anticodons correspond to the most abundant tRNA genes. Sequence homology allows us to propose a function for $\mathbf{4 0}$ of them (Supplementary Table S13). Most are involved in very basic cellular processes: ribosomal proteins, histones, cytoskeleton components, translation elongation factor 2 and succinate dehydrogenase, and many of them have conserved all eight possible copies. The resulting a

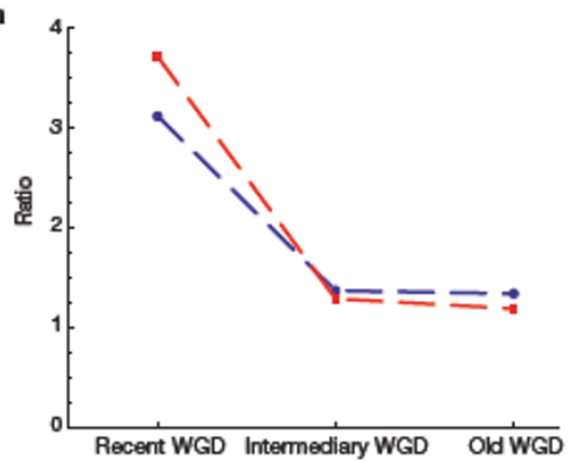

b

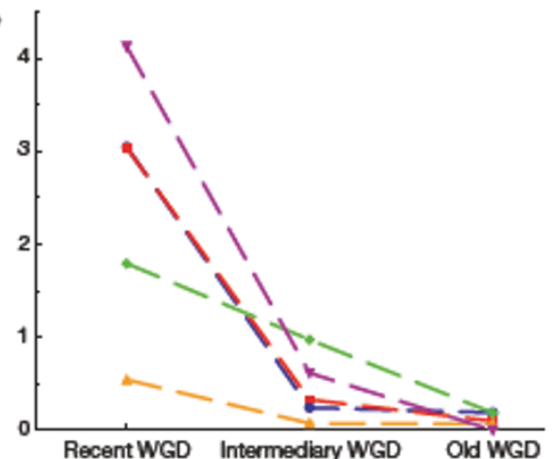

c

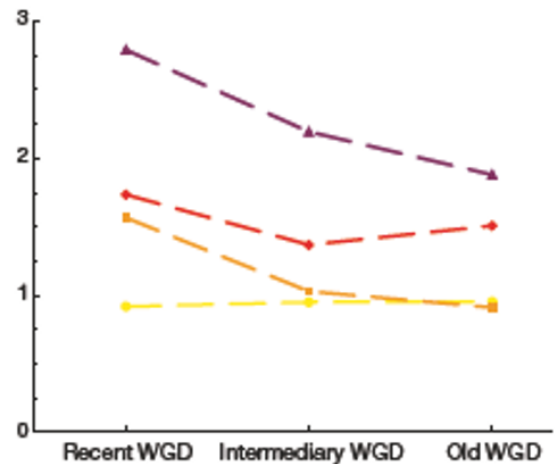

Figure 4 | Retention of duplicated genes according to biological criteria. For each curve, a value of 1 means that the retention of duplicates in one particular category is equal to the mean retention for all the genes. Values below 1 indicate under-retention and values over 1 indicate over-retention. a, Retention rates for proteins forming known complexes in $S$ cerevisiae. Each point represents the ratio of duplicated to non-duplicated genes for genes in complexes in $S$ cerevisiae with orthologues in $P$. tetraur elia, divided by the ratio of total duplicated genes to non-duplicated genes for each duplication. The blue curve is for orthologues of the CYGD list (http:// mips.gsf.de/), and the red curve is for orthologues of ref. 29. b, Retention of gene duplicates in central metabolic pathways: blue, amino-acid metabolism; red, carbohydrate metabolism; green, energy metabolism; orange, lipid metabolism; purple, nudeotide metabolism. Each point represents the ratio of duplicated to non-duplicated genes for genes with unambiguous EC number, divided by the ratio of total duplicated genes to non-duplicated genes for each duplication. c, Expression versus retention across the three WGDs. Each point represents the ratio of duplicated to nonduplicated genes for genes with a defined number of EST matches, divided by the ratio of total duplicated genes to non-duplicated genes for each duplication. Yellow, zero to two EST matches; orange, three to five EST matches; red, six to nine EST matches; purple, ten or more EST matches. 


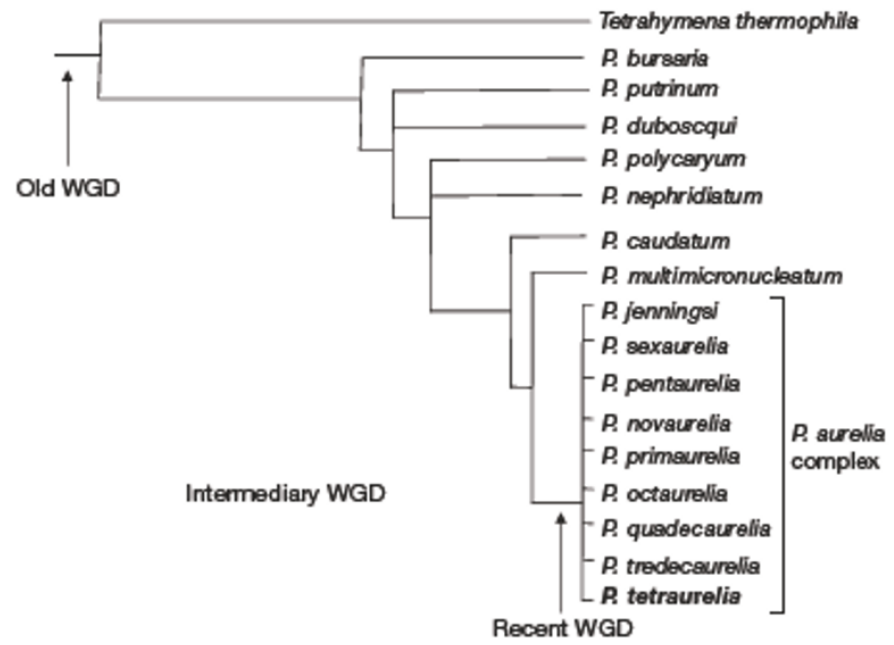

Figure 5 | Dating of genome duplication events. The phylogenetic tree of the Paramexium genus was adapted from ref. 47. Phylogenetic analyses indicate that the old WGD occurred before the divergence of Paramecium and Tetrahymena, and the recent WGD before the divergence of $P$. tetraurelia and $P$. octaurelia. There are currently not enough data to date the intermediary WGD.

relative enhancement of these activities should be a significant effect ofWGD. Indeed, retention of duplicates for some of these genes, such as those encoding ribosome assembly proteins, is also observed in the WGDs of yeast and Arabidopsi 21.22,30. $^{2}$.

\section{Genome duplication and speciation}

To allow dating of the intermediary and old duplications, we sampled 27 gene families for which homologues were found in Tetrahymena thermophila and several outgroup eukaryotes. Phylogenetic analyses indicate that the old duplication may have occurred shortly before the divergence between Tetrahymena and Paramecium lineages, whereas the intermediary duplication is specific to Paramecium (see Supplementary Information). The recent duplication was dated by analysing 12 gene families for which orthologues in species of the
Paramecium genus were available. Phylogenetic trees indicate, with strong bootstrap support, that the recent event occurred after the divergence of $P$. tetraurelia from $P$. caudatum and $P$. multimicronucleatum (Fig. 5). P. tetraurelia belongs to a complex of 15 sibling species (the Paramecium aurelia complex) ${ }^{31}$. Data from other species of the P. aurelia complex indicate that the recent WGD occurred before their divergence from $P$. tetraurelia, which is indicative of a possible link between genome duplication and speciation. Indeed, after a genome duplication, many gene losses occur independently in different populations. This process is therefore expected to lead rapidly to reproductive isolation by Dobzhansky-Muller incompatibility $^{32}$. Interestingly, species of the P. aurelia complex are extremely similar both morphologically and in terms of ecological environment (and hence they were initially thought to correspond to a single species $^{31}$ ). We therefore propose that the explosion of speciation events that gave rise to the $P$. aurelia complex is not the result of adaptative evolutionary events (for example the colonization of new ecological niches) but the neutral consequence of the genome duplication $^{32}$.

\section{WGDs in genome evolution}

Although there is no current consensus on gene fates after a WGD, many current models consider that gene loss is rapid after the duplication, and that functional divergence (either by sub-functionalization or neo-functionalization) can explain most of the retention pattern. The Paramecium data point to another model, which is summarized in Fig. 6. Some genes, representing a fraction of the genome, probably quickly lose their duplicated state, because they can never be detected as duplicates in any of the three WGDs. Duplication of these genes may provide no advantage or may even be counter-selected. However, most of the gene duplicates did not go through this rapid elimination. Their maintenance may be driven at least partly by the maintenance of high expression and stoichiometric constraints. Indeed, many of the duplicates of the recent WGD are functionally redundant. Patterns of retention of more ancient WGDs indicate that most of these duplicates will be progressively lost. Yet the distribution of $K_{d} / K_{\mathrm{s}}$ ratios shows that almost all recent duplicates are under strong purifying selection (Supplementary Fig. S18), indicating that deleterious mutations affecting the coding sequence of one

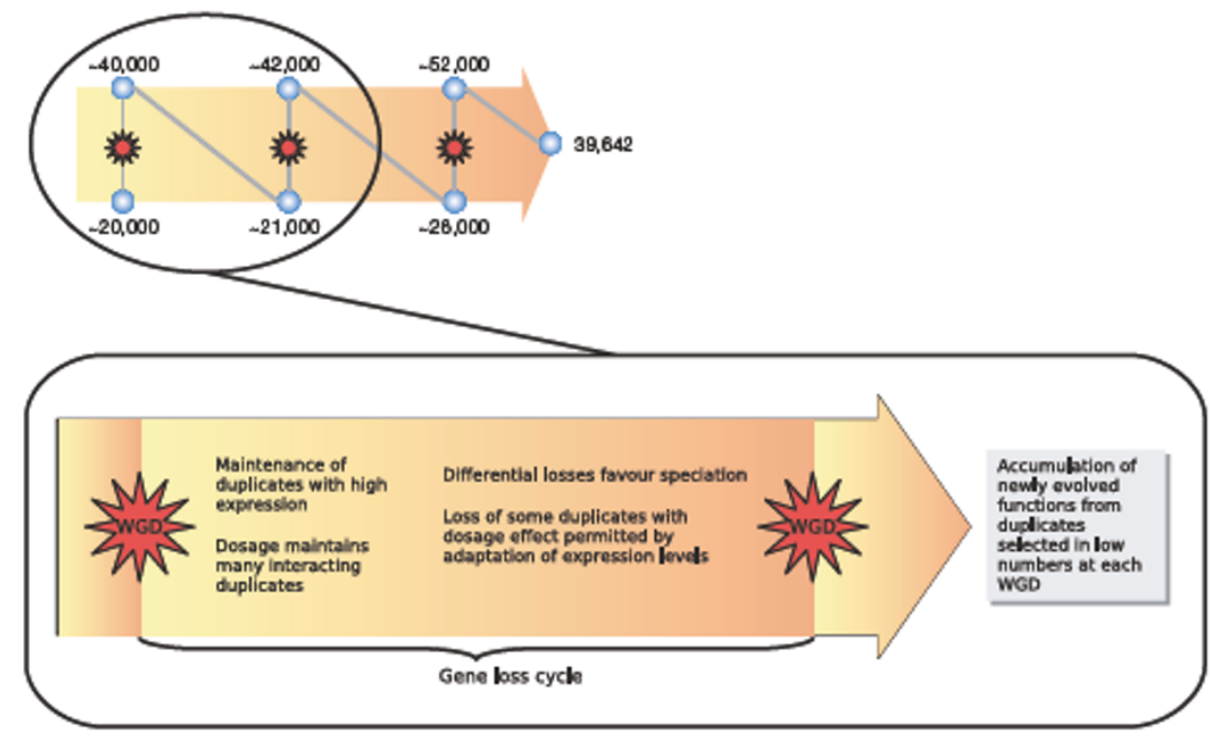

Figure 6 | A model for gradual gene loss after a WGD deduced from differential retention of genes in Paramecium. Top: evolution of gene number through the three WGD events. Estimates of gene numbers at the time of the duplications were made under the simplifying assumption that further geneloss affected only the new duplicates. Bottom: focus on an interWGD period. Just after WGD, genes subject to dosage constraints (that is, genes in complexes or metabolic pathways) and highly expressed genes are preferentially retained as duplicates. Differential loss of paralogues in subpopulations will create barriers to reproduction, favouring speciation. Gradually, the duplicates will erode as the dosa ge effects are compen sated for by new mutations. This massive retention of duplicates across a long evolutionary time enables selection to retain a few new genes with new derived functions. 
gene are not masked by the expression of the other copy. As gene regulation evolves to permit loss without affecting dosage, most genes then gradually resolve to a single state. Another model for duplicate retention favours a buffering effect, in which genes are retained in two copies to provide robustness against mutation for important functions ${ }^{23}$, which would be especially important for a homozygous population. It is worth noting that many plants and Paramecium can undergo self-fertilization and that natural populations of $P$. tetraurelia are probably homozygous most of the time. This model is consistent with high retention for basic physiological processes in Paramecium. However, it is difficult to consider buffering as the main effect of WGD, because it does not explain why so many genes have been retained in many more than two copies.

The appearance of new derived functions may be a secondary product of this basic model. Entire gene modules are retained initially as duplicates with redundant functions, but some of them will evolve to derived ones if there is no need other than stoichiometry for maintaining the redundancy. One example of neo-functionalization is provided by $\delta$ - and $\eta$-tubulins, paralogues of the old WGD, which have distinct functions in the assembly $\left(\delta\right.$-tubulin $\left.{ }^{33}\right)$ or duplication $\left(\eta\right.$-tubulin $\left.{ }^{34}\right)$ of ciliary basal bodies. $\eta$-Tubulin is known only in ciliates, whereas $\delta$-tubulin is conserved in eukaryotes that perpetuate the centriolar structure.

To gain insight into the acquisition of new functions through WGD, we looked for cases of marked sequence divergence between paralogues, because the neo-functionalization model predicts that the two copies will exhibit asymmetric rates of molecular evolution. After correction for multiple testing, we find that $10.9 \%$ of recent duplicates and $16.2 \%$ of intermediary duplicates show asymmetric evolutionary rates. This difference is significant $\left(\chi^{2}=7.55, P<0.01\right.$; see Supplementary Information) and can probably be attributed to the progressive nature of duplicate gene loss: a larger proportion of functionally redundant genes remain among recent duplicates than among the intermediary ones.

Among the 62 intermediary duplicates with asymmetric evolutionary rate, we noticed that the rapidly evolving copy tends to be less retained after the recent WGD than the slowly evolving one ( $26 \%$ versus $66 \% ; \chi^{2}=20, P<10^{-4}$ ). This indicates that neo-functionalized copies might be more prone to pseudogenization at subsequent WGDs.

The sub-functionalization model predicts that agene that has been preserved by sub-functionalization at a given WGD is less likely to be retained in two copies at a subsequent $W_{G D}^{35}$. We observed the opposite pattern: $57 \%$ of genes already retained in the intermediary duplication are retained after the recent WGD, whereas only $47 \%$ of genes not retained after the intermediary duplication are retained after the recent WGD $\left(P<10^{-4}\right)$. Because this might simply reflect different retention rates between functional classes, only genes from the same functional class were considered. We selected families resulting from the ancient duplication for which one member has been retained in two copies after the intermediary WGD but the other has not ( $n=343$ families). In agreement with the prediction of the sub-functionalization model, the frequency of retention after the recent WGD was lower for genes retained after the intermediary WGD than for genes that had lost their duplicate $(60 \%$ versus $67 \% ; \chi^{2}=4.58, P<5 \%$ ). Although significant, this difference is not very strong, which is consistent with the fact that sub-functionalization is an unlikely evolutionary pathway in species with large population sizes ${ }^{35}$.

\section{Conclusion}

The Paramecium duplications have many traits that render them particularly attractive for studying the fates of duplicate genes. Both the high level of gene retention and the strong conservation of synteny helped to define an unprecedentedly large collection of paralogues, with high confidence in their exact origin. In particular, the most recent WGD proved informative on the early evolution of paralogues. We predict a pattem of gene loss that is consistent with progressive decay, acting predominantly at the gene level. The initial maintenance of a complete double set of genes is therefore probably an essential difference between WGD and other large-scale duplications.

We can see parallel evolution for whole protein complexes, metabolic pathways, and known interacting protein groups. All these observations are consistent with a central function of dosage constraints on the evolution of gene duplicates after WGD. We proposea new model for WGD evolution, in which expression and stoichiometry are important in the retention of many duplicate genes, that can thereafter evolve in a few cases towards new derived functions. WGD is therefore fundamentally different from single-gene duplication. Indeed, the gene categories that participate in these two duplication mechanisms are significantly different ${ }^{22}$. The fact that WGDs have been postulated or demonstrated at important diverging points in eukaryotic evolution ${ }^{28.36-38}$ makes plausible the direct link between these amplifications and the appearance of some eukaryote-specific novelties. Paramecium supports different mutational systems for genetic analysis, including the use of RNA-mediated interference, that can be implemented at the genome scale. We expect that the present study will lead to further work exploring the functional fate of WGDs in detail.

\section{METHODS}

Sequencing and assembly. The whole-genome shotgun and assembly method is described in detail in Supplementary Information.

Gene annotation. Protein-coding genes were predicted by combining $a b$ initio modds, Paramecium cDNA alignments, and alignments of proteins and genomic DNA from other species. The integration of the data was realized using $\mathrm{GAZE}^{n}$. Details are given in Supplementary Information.

Genome duplication. A global all-against-all comparison of every predicted protein was performed with the Smith-Waterman algorithm $(e$ value $<0.1$ ) and a set of 12,504 best reciprocal hits (BRHs) based on alignment scores, Each scaffold was scanned with a sliding window of BRHs with window size (w) of 10 , and $60 \%$ of genes were paired (parameter $p$ ). Paralogous blocks were obtained with this windowing strategy and by merging contiguous windows associated with a common target scaffold. In the next step, 1,477 non-BRH syntenic matches found among the ten best matches of any protein with no BRH were added to the paralogue list. These paralogons were fused to create a single molecule with the assumption that every gene without a paralogue corresponds to a gene whose paralogue was lost after the duplication. This creates a succession of genes approximating the gene order of the pre-duplication region, which we called an ancestral block.

Todescribe the older duplications, the same approach was iterated three times with the following parameters: intermediary WGD, $w=10$ and $p=41 \%$; old WGD and ancient duplicates, $w=20$ and $p=30 \%$. The paralogous links for each duplication are presented in Supplementary Tables S17-S20, and the composition of the ancestral blocks is given in Supplementary Table 21 . Figure 2 was constructed using Circos (http://mkweb.bcgsc.ca/circos).

Evolution of genes and non-coding sequences. $K_{\mathrm{a}}$ and $K_{\mathrm{a}}$ values were calculated on the entire set of paralogues for each WGD, using the codeml software from the PAML package ${ }^{40}$.

Analysis of metabolic pathways. We used PRIAM ${ }^{41}$ to identify 5,617 genes containing at least one Enzyme Commission (EC) number. They were remapped on to metabolic pathways by using the KEGG database"

Protein complexes. We remapped Saccharomyces cerevisiae complexes (ref. 29 and http://mips.gsf.de/) on to the Paramecium proteome by using orthologous links (see Supplementary Information). A protein-protein interaction was considered conserved if each protein had an orthologue in the Parameciumgenome, and a protein complex was considered conserved if it contained at least one conserved protein-protein interaction. From the 1,602 complexes of the MIPS catalogue and the 422 complexes from ref. 29, we found respectively 443 and 61 conserved complexes in the paralogons of the old WGD.

Phylogeny of the WGDs. Phylogenetic trees were inferred from protein sequence alignments by maximum likelihood. We used Phym $\mathrm{r}^{\mathrm{s}}$ under the JTT model of protein evolution ${ }^{4}$, with site-to-site rate variation modelled on a discrete $\gamma$ distribution (four categories, shape parameter $\alpha$ and proportion of invariable sites estimated from the data). The robustness of the phylogenetic inference was estimated by bootstrap (500 replicates). Phylogenetic trees are 
available as Supplementary Information. Data on the Tetrahymena proteinswere from The Institute for Genomic Research (http:/www.tigr.org/tdb/e $2 \mathrm{k} 1 / \mathrm{ttg} /$ ).

Received 4 May 2006; accepted 31 August 2006.

Published online 1 November 2006.

1. Jahn, C. L.\& Klobutcher, L. A. Genome remodeling in ciliated protozoa. Annu. Rev. Microbiol. 56, 489-520 (2002).

2. Betermier, $M$. Large-scale genome remodel ling by the developmentally programmed elimination of germ line sequences in the ciliate Paramecium. Res. Microbiol. 155, 399-408 (2004).

3. Le Mouel, A. Butler, A. Caron, F. \& Meyer, E. Developmentally regulated chromosome fragmentation linked to imprecise elimination of repeated sequences in paramecia. Eukaryot. Cell 2, 1076-1090 (2003).

4. Sonneborn, T.M. in Handbook of Genetics (ed. King, R.) 469-594 (Plenum, New York, 1974).

5. Jaffe, D. B. et $d$. Whole-genome sequence assembly for mammalian genomes: Arachne 2. Genome Res. 13, 91-96 (2003).

6. Zagulski, M. et $a$. High coding density on the largest Paramecium tetraurelia somatic chromosome. Curr. Biol. 14, 1397-1404 (2004).

7. Bhattacharya, D, Yoon, H. S. \& Hackett, J. D. Photosynthetic eukaryotes unite: endosymbiosis connects the dots. Bioessays 26, 50-60 (2004).

8. Vision, T. L, Brown, D. G. \& Tanksley, S. D. The origins of genomic duplications in Arabidopsis. Science 290, 2114-2117 (2000).

9. Blanc, G, Hokamp, K. \& Wolfe, K. H. A recent polyploidy superimposed on older large-scale duplications in the Arabidopsis genome. Genome Res 13, 137-144 (2003).

10. Bowers, L. E, Chapman, B. A., Rong, J. \& Paterson, A. H. Unravelling angiosperm genome evolution by phylogenetic analysis of chromosomal duplication events. Nature 422, 433-438 (2003).

n. Dietrich, F. S. et al. The Ashbya gossypii genome as a tool for mapping the ancient Saccharomyces cerevisiae genome. Science 304, 304-307 (2004).

12. Dujon, B. et al. Genome evolution in yeasts. Nature 430, 35-44 (2004).

B. Jaillon, $\mathrm{O}$. et al. Genome dup lication in the teleost fish Tetraodon rigroviridis reveals the early vertebrate proto-karyotype. Nature 431, 946-957 (2004).

14. Kellis, M, Birren, B.W. \& Lander, E. S. Proof and evolutionary analysis of ancient genome duplication in the yeast Saccharomyces cerevisiae Nature $428,617-624$ (2004).

15. Simillion, C., Vandepoele, K., Van Montagu, M. C, Zabeau, M. \& Van de Peer, Y. The hidden duplication past of Arabidopsis thaliana. Proc. Natl Acad. Sci. USA 99, 13627-13632 (2002).

16. Wolfe, K. H. \& Shields, D. C. Molecular evidence for an ancient dup lication of the entire yeast genome. Nature 387, 708-713 (1997).

17. Yu, $\perp$ et al. The genomes of Oryza sativa a history of dup lications. PL $\alpha S$ Bial. 3, e38 (2005).

18. Dubrana, K. \& Amar, L. Programmed DNA under-amplification in Paramecium primaurelia. Chromosoma 109, 460-466 (2000).

19. Berger, J. D. \& Schmidt, H. J. Regulation of macronudear DNA content in Paramecium tetraurelia. 1 Cell Biol. 76, 116-126 (1978).

20. Andalis, A. A. et $a$. Defects arising from whole-genome dup lications in Saccharomyces cerevisiae Genetics 167, 1109- 1121 (2004).

21. Blanc, G. \& Wolfe, K. H. Functional divergence of duplicated genes formed by polyploidy during Arabidopsis evolution. Plant Cell 16, 1679-1691 (2004).

22. Maere, S. et al. Model ing gene and genome duplications in eukaryotes. Proc. Natl Acad Sci. USA 102, 5454-5459 (2005).

23. Chapman, B. A. Bowers, L E, Feltus, F. A. \& Paterson, A. H. Buffering of crucial functions by paleologous duplicated genes may contribute cyclicality to angiosperm genome duplication. Proc. Natl Acad Sci. USA 103, 2730-2735 (2006).

24. Papp, B., Pal, C. \& Hurst, L. D. Dosage sensitivity and the evolution of gene families in yeast. Nature 424, 194-197 (2003).

25. Kondrashov, F. A., Rogozin, L. B., Wolf, Y. I. \& Koonin, E. V. Selection in the evolution of gene duplications. Genome BioL. 3, RESEARCHOOO8 (2002).

26. Lynch, M.\& Katju, V. The altered evolutionary trajectories of gene duplicates. Trends Genet. 20, 544-549 (2004).
27. Veitia, R. A. Paralogs in polyploids one for all and all for one? Plant Cell 17, 4-11 (2005).

28. Ohno, S. Evolution by Gene Duplication (Allen \& Umwin, London, 1970).

29. Gavin, A. C. et $a$. Proteome survey reveals modularity of the yeast cell machinery. Nature 440, 631-636 (2006).

30. Seoighe, C.\& Wolfe, K.H. Yeast genome evolution in the post-genome era. Curr. Opin. Microbiol. 2, 548-554 (1999).

31. Coleman, A. W. Paramecium aurelia revisited. L. Eukaryot. Microbiol. 52, 68-77 (2005).

32. Scannell, D. R, Byrne, K. P , Gordon, J. L., Wong, S. \& Wolfe, K. H. Multiple rounds of speciation associated with reciprocal gene loss in polyploid yeasts. Nature 440 341-345 (2006).

33. Garreau de Loubresse, N. Ruiz, F, Beisson, J. \& Klotz, C. Role of delta-tubulin and the C-tubule in assembly of Paramecium basal bodies. BMC Cell Bial. 2, 4 (2001).

34. Ruiz, F. et $d$. The SM19 gene, required for duplication of basal bodies in Paramecium, enco des a novel tubulin, $\eta$-tubul in. Curr. Biol. 10, 1451-1454 (2000).

35. Force, A. et al. Preservation of duplicate genes by complementary, degenerative mutations. Genetics 151, 1531-1545(1999).

36. Dehal, P.\& Boore, J.L. Two rounds of whole genome duplication in the ancestra vertebrate. PLOS Biol. 3, e314 (2005).

37. Postlethwait, J, Amores, A, Cresko, W, Singer, A.\& Yan, Y. L. Subfunction partitioning, the teleost radiation and the annotation of the human genome. Trends Genet. 20, 481-490 (2004).

38. De Bodt, S, Maere, S. \& Van de Peer, Y. Genome duplication and the origin of angiosperms. Trends Ecol. Evol. 20,591-597 (2005).

39. Howe, K. L., Chothia, T. \& Durbin, R. GAZE: a generic framework for the integration of gene-prediction data by dynamic programming Genome Res. 12, 1418-1427(2002).

40. Yang, Z. PAML: a program package for phylogenetic analysis by maximum likelihood. Comput. Appl. Biosci. 13, 555-556 (1997).

41. Claudel-Renard, C, Chevalet, C, Faraut, T.\& Kahn, D. Enzyme-specificprofiles for genome annotation: PRIAM. Nucleic Acids Res. 31, 6633-6639 (2003).

42. Kanehisa, M. Goto, S, Kawashima, S., Okuno, Y. \& Hattori, M. The KEGG resource for deciphering the genome. Nucleic Acids Res. 32, D277-D280 (2004).

43. Guindon, S. \& Gascuel, O. A simple, fast, and accurate algorithm to estimate large phylogenies by maximum likelihood. Syst. Biol. 52, 696-704 (2003).

44. Jones, D. T. Taylor, W. R. \& Thornton, J.M. The rapid generation of mutation data matrices from protein sequences. Comput. Appl. Biosci. 8, 275-282 (1992).

45. Eichinger, L. et al. The genome of the social amoeba Dictyostelium discoideum Nature 435, 43-57 (2005).

46. Matsuzaki, M. et al. Genome sequence of the ultrasmall unicellular red alga Cyanidioschyzon merolae 10D. Nature 428, 653-657 (2004).

47. Fokin, S. I. et al. Morphological and molecular investigations of foramecium schewiakoffi sp. nov. (Ciliophora, Oligohymenophorea) and current status of distribution and taxonomy of Poramecium spp. Eur. L Protist. 40, 225-243 (2004).

48. Keeling P. J.et al. The tree of eukaryotes. Trends Ecol. Evol. 20, 670-676(2005).

Supplementary Information is linked to the online version of the paper at wwwnature.com/nature.

Acknowledgements We thank P. Y. Bourguignon for help in statistical analyses. This work has been funded by the Consortium National de Recherche en Génomique, the CNRS, the Polish Ministry of Science and the Agence Nationale de la Recherche.

Author Information The final assembly and annotation are deposited in the EMBL/ Genbank/DDBJ databases under accession numbers CT867985-CT868681. An annotation browser and further information on the project are available from http://www.genoscope.cns.fr/paramecium and

http:/paramecium.cgm.cnrs-gif.fr. Reprints and permissions information is available at www.nature.com/ reprints. The authors declare no competing financial interests. Correspondence and requests for materials should be addressed to P.W. (pwincker@genoscope.cns.fr). 